\title{
Analysis of Two Vibrating Suction Methods ${ }^{*}$
}

\author{
Kun Wang, Wei Wang, Dazhai Li \\ and Guanghua Zong \\ Robotics Institute \\ Beihang University \\ Beijing 100083, China \\ wangkunggg@163.com
}

\author{
Houxiang Zhang, Jianwei Zhang \\ TAMS, Department of Informatics \\ University of Hamburg \\ Vogt-Kölln-Straße 30, Hamburg \\ 22527, Germany \\ hzhang@informatik.uni-hamburg.de
}

\author{
Zhicheng Deng \\ Nokia Research Center \\ Nokia (China) Investment CO.,LTD. \\ 100176,Beijing, China \\ ext-zhicheng.deng@nokia.com
}

\begin{abstract}
In this paper, a novel vibrating method based on the principle of vibrating suction method is presented, which is called pulse vibrating method. To discuss this method in depth and evaluate its performance, simplified mathematical model based on some assumptions for both the previous sin vibrating method and the pulse vibrating method are built, and a new experimental platform is developed as well to verify the validity of the mathematical models. The experiments indicate that the experiments meet the mathematical model with only small deviation caused by some unknown factors. The experimental results also show that the pulse vibrating method is much better than the sin vibrating method for higher negative air pressure and less power consumption. At the end of this paper, conclusion is given and future work is proposed to further analyze the principle of the vibrating suction method.
\end{abstract}

\section{Index Terms - Vibrating suction method, Suction cup, Climbing robot}

\section{INTRODUCTION}

Mini-climbing robots are regarded as useful and safe equipments for infrastructures inspection, urban search and civil exploration and so on for its portability, costeffectiveness and less potential dangers [1] [2]. By far, there are 3 adsorption methods for mini-climbing robots to attach to the working surfaces: magnetic adsorption [3][4], vacuum adsorption[5][7] and molecular adsorption[6]. Magnetic adsorption method can only be used on ferromagnetic surface, so that its application fields are very limited. Molecular adsorption method, which is still on the development stage, is also very subject to the material of the working surface and the environment. Based on these considerations, vacuum adsorption method is widely applied for the mini-climbing robots.

The vacuum adsorption method includes active adsorption [7][8] and passive absorption[9][10]. The active adsorption produces negative air pressure in suction cup by a vacuum device. Though it can produce high negative air pressure and large absorption force, it needs a long trachea [11], which makes the robot very heavy. While the passive absorption does not need a vacuum device and a long trachea. It produces negative air pressure by expanding the volume of the suction cup. By utilizing this method, the climbing robots can be designed much smaller and lighter than the robots using active adsorption [9][10]. Of course, the passive adsorption has some shortcomings such as relatively low negative air pressure, poor safety. Moreover, it can not stick to the wall for a long time.

In this paper, a novel vibrating suction method is presented. To discuss this method in depth, simplified mathematical models for both the previous sine vibrating method and the pulse vibrating method are built, and also a new experimental platform is developed to verify the validity of the mathematical models. The experimental results tell as well that the pulse vibrating method is much better than the sine vibrating method for higher negative air pressure and less power consumption. At the end of this paper, conclusion is given and future work is proposed to further analyze the principle of the vibrating suction method.

\section{RELATED WORK}

In our previous paper [12], vibrating suction method is proposed to establish the vacuum in the suction cup by vibrating the cup with respect to the wall. Preliminary principle of the vibrating suction method is analyzed, and the validity of the analysis is verified through an experimental platform. The experimental platform and its schematic are shown in Fig. 1.

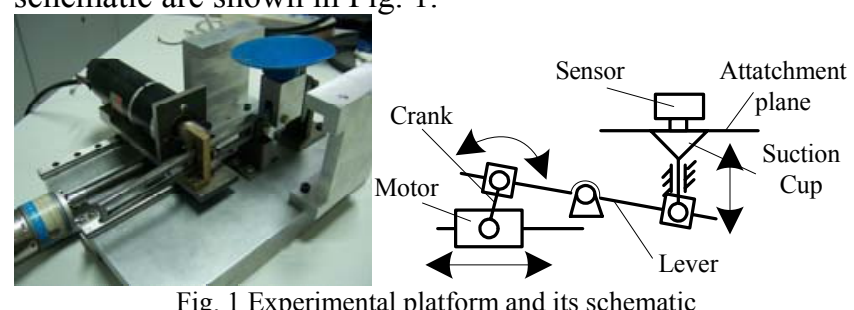

Fig. 1 Experimental platform and its schematic

As shown in the Fig.1, the motor drives the crank in continuous rotation, which is then converted into up and down motion of the suction cup via the lever mechanism, and make the suction cup vibrate on the attachment plane. The air pressure in the suction cup can be sampled through the sensor. According to the experiments, the following conclusions could be concluded.

\footnotetext{
* This work is supported by a grant from the National High Technology Research and Development Program of China (863 Program No.2006AA04Z250) .

* This work is partially supported by the "National Natural Science Foundation of China" (No.50605501).
} 
1) The vibrating suction method can produce stable negative air pressure with a certain vibration amplitude level and vibration frequency.

2) The time of the suction cup between natural state and the state of producing stable negative air pressure is very short $(<1 \mathrm{~s})$, which means the response speed is fast.

3) The larger the amplitude and frequency are, the higher the negative air pressure in suction cup will be achieved. On the contrary, the smaller the amplitude and frequency are, the lower the negative air pressure is.

4) The vibration amplitude plays a more important role than the vibration frequency in producing the negative air pressure.

In the previous work, we designed two mini-climbing robots [9][13][14], which make use of the principle of vibrating suction method. They can adsorb on rough wall. The experiments are shown in Fig. 2.

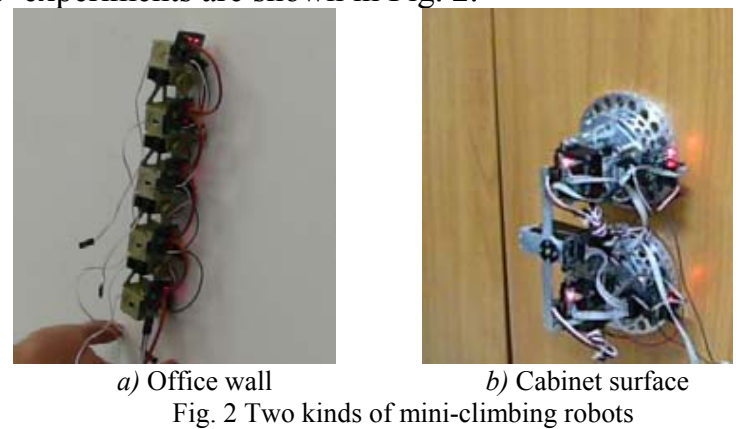

However, there are also some major drawbacks about the experimental platform.

1) The dimension error of the structure is too large, which lowers the precision of acquired data.

2) The range of the amplitude is too small, which is from $0.6 \mathrm{~mm}$ to $1.2 \mathrm{~mm}$, and can not give enough guidance to applications.

3) The sensor can only acquire negative data. But according to the analysis, there should be positive data.

\section{MATHEMATICAL MODEL OF TWO VIBRATING METHODS}

Silicone rubber suction cups have high seal performance on glass planes [12], so the leakage air flow could be ignored within a short period(less than $5 \mathrm{~s}$ ). To simplify the evaluation process of the two vibrating methods, glass plane is adopted in the experimental platform, therefore, we can suppose that the quality of the air in the suction cup is identical to the quality of the air in the suction cup which is max compression, whenever expanding or compressing. But if the attachment plane is not glass, the assumption above is wrong, because the leakage can not be ignored.

In addition, another assumption should be presented. That is the air in the suction cup has to meet the following equation.

$$
P_{i} V=m R T_{k}
$$

This is one of the classical equations in the thermodynamics. It established the relationship between the pressure $(P i)$ of the air in the suction cup and the volume $(V)$ of the suction cup with a certain mol. The variation of the temperature is ignored. And $R=8.31, T k=300 \mathrm{~K}$.

\section{A. Sine vibrating method}

The sine vibrating method could be achieved very easily. Fig. 3 shows the schematic of this method.

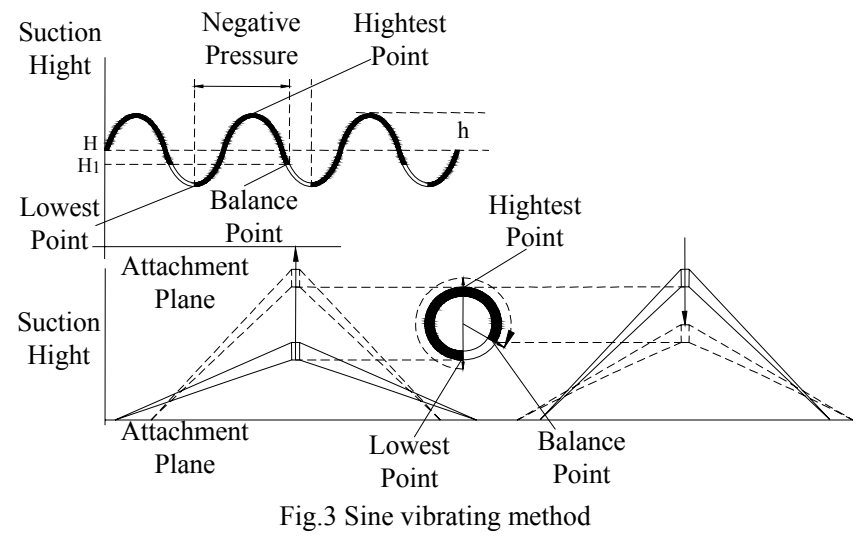

$H_{1}$ in Fig. 3 is the air pressure balance height of the suction in vibrating. $H$ in Fig. 3 is the air pressure balance height of the suction in stillness. Assume that the air pressure in the suction cup is $P_{0}$, which is identical to atmospheric pressure. Then equation (2) can be obtained through equation (1).

$$
P_{0} \cdot \frac{1}{3} \pi R_{S}^{2}(H-h)=m R T_{k}
$$

In equation (2), $R s$ is the radius of the suction cup, $H$ is the height of the suction cup in natural state, $h$ is the amplitude. Assume that, in the sine waveform vibrating state, the air pressure in the suction cup is $P_{s}$. Then equation (3) can be denoted by equation (1).

$$
P_{s} \cdot \frac{1}{3} \pi R_{S}^{2}(H+h \sin \varpi t)=m R T_{k}
$$

In equation (3), $\varpi$ is the angular velocity of the rotation movement, $t$ is the time. The $P_{s}$ can be obtained via equations (2) and (3).

$$
P s=\frac{P_{0}(H-h)}{H+h \sin \varpi t}
$$

For a period of time $T$, assume the average air pressure in the sine waveform vibrating state is $\overline{P_{S}}$. Then the $\overline{P_{S}}$ could be denoted by equation (5).

$$
\overline{P S}=\frac{\int_{0}^{T} P s d t}{T}=\frac{P_{0}(H-h)}{T} \int_{0}^{T} \frac{1}{H+h \sin \varpi t} d t
$$

\section{B. The pulse vibrating method}

Negative air pressure can be produced by vibrating the suction cup [12], and is related to the extent of the changing volume of the suction cup. The sine vibrating method, as shown in Fig.3, always focuses on the $H$ by $h$. The maximum height of the suction cup is $H+h$, which occurs instantaneously. While, in other times, the height of the suction cup is much smaller than $H+h$, therefore, the volume of the suction cup is not taken full advantage of. To improve this situation, pulse vibrating method is proposed, which pushes the suction cup rapidly to the lowest point to achieve 
the biggest compression rate, and then pulls it rapidly to the highest point to achieve the biggest expansion rate, after that, hold the suction cup at the highest point for a certain period, which is decided according to the roughness of the attachment plane. Until now, a complete cycle of pulse vibration is obtained. Fig. 4 shows the schematic.

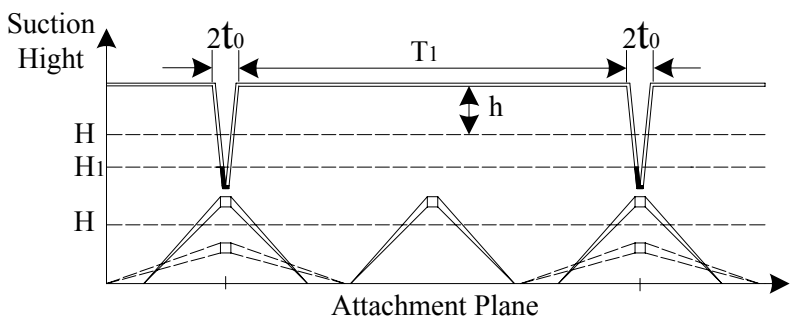

Fig.4 Pulse vibrating method

In Fig.4, $T_{I}$ is the hold time; and both the pushing time and the pulling time is $t o$. All the other parameters are the same as the sine vibrating method. So anytime, the air pressure in the suction cup in the pulse vibrating state $P_{p}$, can be expressed by equations (6) and (7).

$$
\begin{aligned}
& P_{p} \cdot \frac{1}{3} \pi R_{S}^{2}[H+\tilde{h}(t)]=m R T \\
& \tilde{h}(t)=\left\{\begin{array}{l}
\left.h-\frac{2 h}{t_{0}} \cdot t \ldots \ldots \ldots \ldots \ldots . . . . \ldots, t_{0}\right) \\
\frac{2 h}{t_{0}}\left(t-t_{0}\right)-h \ldots \ldots \ldots\left[t_{0}, 2 t_{0}\right) \\
h \ldots \ldots \ldots \ldots \ldots \ldots\left[t_{0},\left(T_{1}+2 t_{0}\right)\right]
\end{array}\right.
\end{aligned}
$$

The above equation (7) is the pulse function. Then equation (8) can be available by equations (2), (6) and (7).

$$
P_{p}=\frac{P_{0}(H-h)}{H+\widetilde{h}(t)}
$$

For a period of time $T$, which is same with the $T$ in equation (5), the average air pressure in the sine waveform vibrating state is $\overline{P_{p}}$, which can be given by equation (9).

$$
\overline{P_{p}}=\frac{\int_{0}^{T} P_{p} d t}{T}=\frac{P_{0}(H-h)}{T}\left(\frac{t_{0}}{h} \ln \frac{H+h}{H-h}+\frac{T-2 t_{0}}{H+h}\right)
$$

C. Comparison of the two vibrating methods

The mathematical model of the two vibrating methods above is under ideal state. There are still many parameters can not be determined, such as the speed of the air flow in/out of the suction cup, the Young's modulus of the suction cup, energy loss caused by the friction between the suction cup and the attachment plane and the shape transformation of the suction cup.

Ratio $k$ is proposed in order to minimize the impact of the unknown factors. Because the impact is exist in both of the two vibrating methods. And the errors of the impact of the unknown factors to the two vibrating methods are same. Therefore it can eliminate the unknown factors and the same errors, so the result is closer to the real value. Ratio $k$ is defined as equation (10).

$$
k=\frac{\overline{P_{p}}}{\overline{P_{s}}}=\left(\frac{t_{0}}{h} \ln \frac{H+h}{H-h}+\frac{T-2 t_{0}}{H+h}\right) /\left(\int_{0}^{T} \frac{1}{H+h \sin \varpi t} d t\right)
$$

\section{EXPERIMENT}

\section{A. Experimental platform and its schematic}

We designed an experimental platform to verify the above mathematical model. The photo of the experimental platform and its schematic are shown in Fig. 5 and Fig. 6.

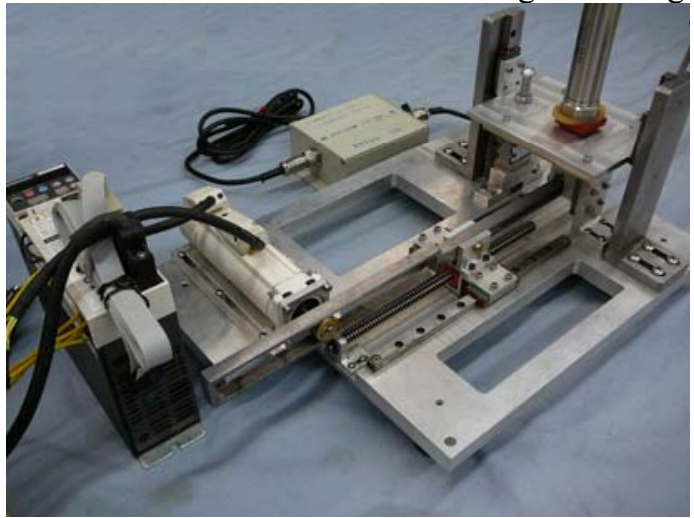

Fig. 5 The photo of the experimental platform

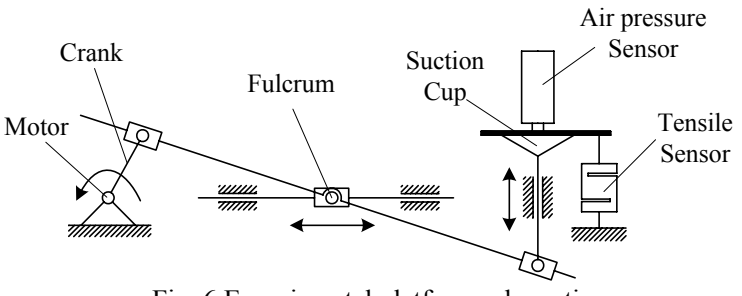

Fig. 6 Experimental platform schematic

As shown in the Fig.6, the motor drives the crank in continuous rotation, then the crank transfers the movement to suction cup through a lever mechanism and make the suction cup vibrate on the plane. The air pressure in the suction cup and the attachment force can be acquired by the two sensors, and send to a PC. Compared with the previous experimental platform shown in Fig 1, this experimental platform has the following advantages.

1) Precise position and velocity control capability. By using digital AC servo motor, precise position and velocity could be achieved.

2) Wide range amplitude. The range of the amplitude varies from $0.5 \mathrm{~mm}$ to $10 \mathrm{~mm}$, which is wider than that of the previous one, and it can be adjusted by changing the position of the fulcrum.

3) Large-range air pressure sensor is adopted, which can measure plus air pressure. Make the experimental data more complete.

4) The use of the force sensor is more reasonable.

\section{B. Experimental data acquisition}

Experiments for the two vibrating methods are carried out under the same conditions in order to verify the validity of the mathematical model and improve the insufficiency of the previous experimental data.

There are 4 variable parameters in the experiments. When one of them varied, the others remain unchanged. The 4 parameters of these two vibrating methods are listed in TABLE I. 
TABLE I

PARAMETERS IN EXPERIMENTS

\begin{tabular}{|c|c|l|c|}
\hline Order & \multicolumn{1}{|c|}{ Parameter } & \multicolumn{1}{|c|}{ Performances } & unit \\
\hline \multirow{2}{*}{1} & frequency for sine & $1,2,5,10$ & $\mathrm{~Hz}$ \\
\cline { 2 - 4 } & frequency for pulse & $1,2,4,8$ & $\mathrm{~s}$ \\
\hline 2 & amplitude & $0.5,1.0,1.5,2.0,2.5,3.0$ & $\mathrm{~mm}$ \\
\hline 3 & suction cup diameter & $30,40,70$ & $\mathrm{~mm}$ \\
\hline \multirow{2}{*}{4} & attachment plane & $\begin{array}{l}\text { glass, aluminum, wood, wall, } \\
\text { wallpaper }\end{array}$ & \\
\hline
\end{tabular}

\section{RESULTS ANALYSIS AND SIMULATION}

\section{A. Comparison of the two vibrating methods}

Two distinct groups of data from large amounts of experimental data are selected for comparison. Simulations, in which the attachment plane is glass and the diameter of the suction cup is $40 \mathrm{~mm}$, are shown from Fig.7 to Fig.10. In these figures, the upper horizontal line represents the atmospheric pressure, and the lower horizontal line represents the average air pressure in suction cup.
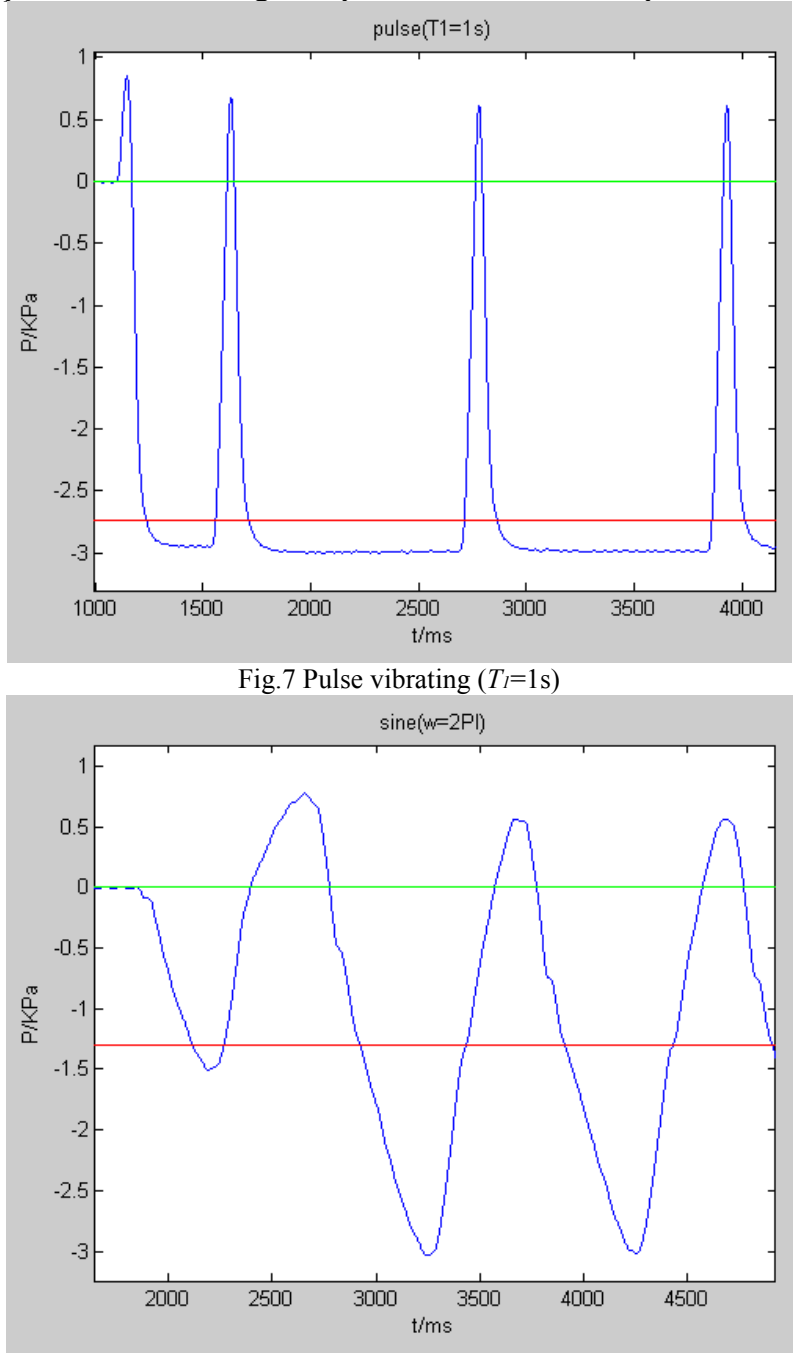

Fig.8 Sine vibrating $(\varpi=2 \pi)$

The parameters of the Fig.7 and Fig. 8 are $H=3 \mathrm{~mm}$, $h=2.5 \mathrm{~mm}, \varpi=2 \pi, T_{l}=1 \mathrm{~s}$ and $t_{0}=0.1 \mathrm{~s}$. So we can calculate the theoretical result $k=0.41$ via equation (10), and obtain the experiment $k=0.47$ through experimental data. Basically speaking, the experiment results meet the mathematical model considering some unknown factors.

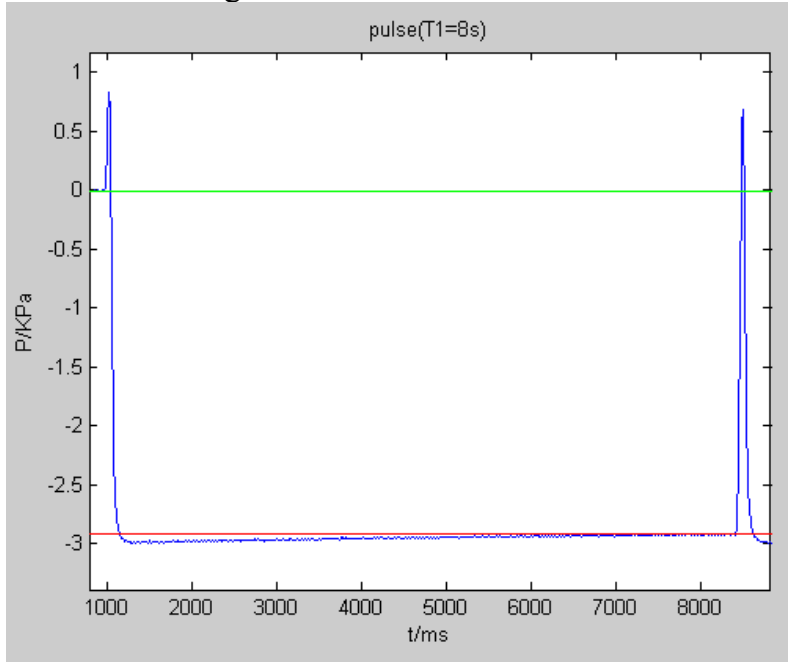

Fig.9 Pulse vibrating $\left(T_{l}=8 \mathrm{~s}\right)$

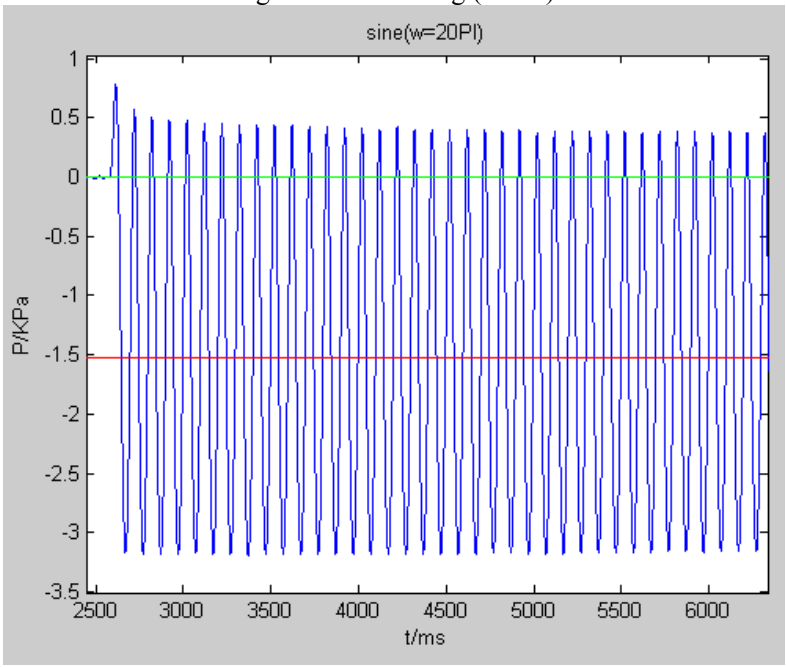

Fig.10 Sine vibrating $(\varpi=20 \pi)$

The parameters of the Fig. 9 and Fig. 10 are $H=3 \mathrm{~mm}$, $h=2.5 \mathrm{~mm}, \varpi=20 \pi, T_{l}=8 \mathrm{~s}$ and $t_{0}=0.1 \mathrm{~s}$. Through equation (10), we get $k=0.31$. While the $k$ obtained by experimental data is 0.51 . Hence, for the $k$ value, the theoretical result has great deviation from the experimental result. That means, the impact of the unknown factors can not be ignored in case that $T_{l}$ is too large. The experiment also shows that the larger $T_{l}$ is, the more leakage will be. And the average air pressure in suction cup is greatly affected by the $T_{l}$ value.

$B$. The overall trend analysis

The average air pressure with glass attachment plane in suction cup of diameter $40 \mathrm{~mm}$ was calculated. The 3-D images are shown as Fig.11 and Fig.12. 


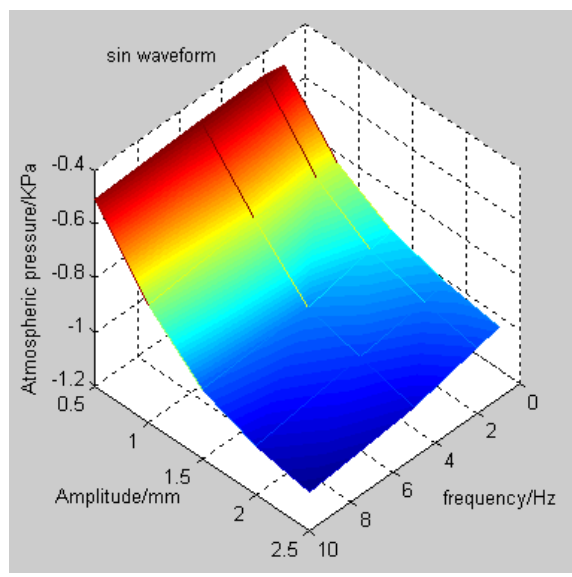

Fig.11 Sine vibrating

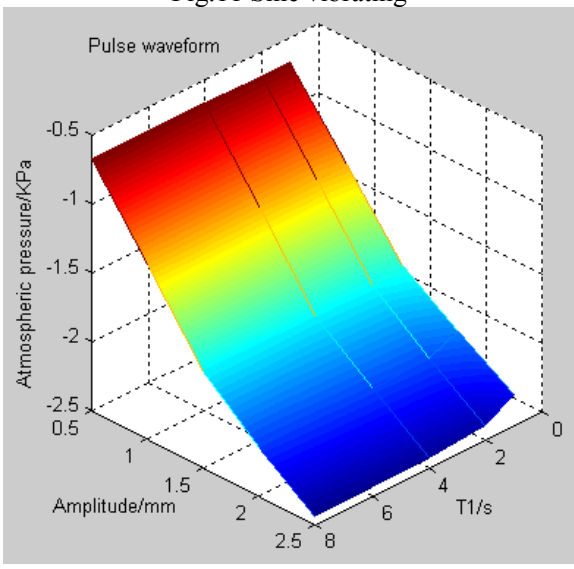

Fig.12 Pulse vibrating

The two above figures show that the average negative air pressure is increasing with the increasing amplitude, frequency and hold time $T_{l}$. But the effect of the three parameters to the average negative air pressure is different. With the increasing amplitude, the increasing trend of average negative air pressure is obviously. Though the increasing frequency and the hold time $T_{l}$ can also make the average negative air pressure increase, the trend is not as obvious as the increasing amplitude. Through these experiments, the following conclusions could be made.

1) Increasing amplitude can increase the average negative air pressure in suction cup effectively for both the two vibrating methods.

2) Increasing frequency can increase the average negative air pressure in suction cup for the sine vibrating method with greater energy consumption.

3) Increasing the hold time $T_{l}$ can increase the average negative air pressure in suction cup for the pulse vibrating method with less energy consumption.

4) The pulse vibrating method can produce much higher average negative air pressure in suction cup than the sine vibrating method with the same energy consumption.

The forth conclusion about energy consumption can't be achieved in mathematical models now, because it is made through the experiment. More details will be discussed in future work.

\section{FUTURE WORK}

\section{A. Active area of the suction cup}

The active area of the suction cup [15] is proposed for further analyzing the principles of the vibrating suction methods, which produces the negative air pressure by vibrating suction cups. As shown in Fig.13, the attachment area of the silicone rubber suction cup will change when the cup is compressed.

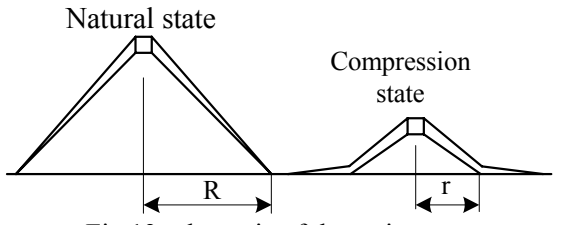

Fig.13 schematic of the active area

The left suction cup is in natural state, and its attachment radius is $R$. The right suction cup is in compression state, and its attachment radius is $r$. The two radiuses are different, which makes the air pressure and the attachment force different. To improve the vibrating suction method principle, the following two points will be conducted in future work.

1) Introducing the active area of the suction cup to the mathematical model.

2) Analyzing the attachment force of the vibrating suction cup by utilizing the active area.

3) The energy consumption of the two vibrating methods.

\section{B. The attachment module based on pulse vibrating}

Experiment shows that the pulse vibrating method can produce higher negative air pressure than the sine vibrating method, so does the attachment force. Therefore, we proposed a new attachment module based on pulse vibrating. The schematic is shown in Fig.14.

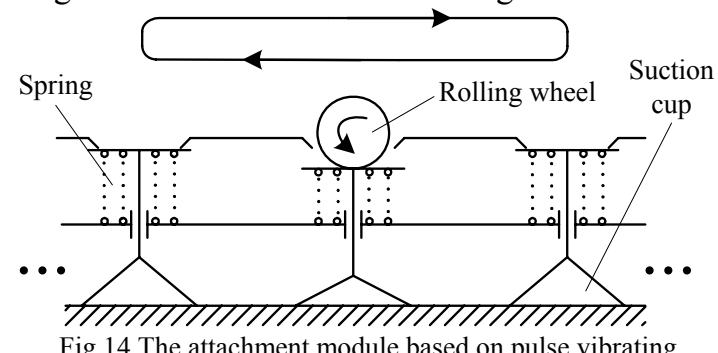

As the figure shows, when the rolling wheel comes to a suction cup, the suction cup will be compressed rapidly. When the rolling wheel passes through the suction cup, the suction cup will be expanded rapidly by the spring. Then the rolling wheel will come to the next suction cup. The expanding suction cup will hold the expanding state until the rolling wheel comes next time. Then, a cycle is finished. Every suction cup will be compressed, expanded and held the expanding state, then the pulse vibrating come true. The hold time $T_{l}$ can be adjusted by changing the speed of the rolling wheel, but the feasibility of it needs further verification. 


\section{CONCLUSION}

This paper analyses the impact of the two vibrating methods to the air pressure of the suction cup, verifies the mathematical model by experiments. The following conclusions could be made through the experiments and the discussions.

1) Pulse vibrating method is better than sine vibrating method, but complex mechanical structure is needed.

2) The three parameters to the order of importance is amplitude, holding time and frequency.

3) The active area of the suction cup is presented to analyse the principle of vibrating suction method.

4) A new attachment module based on pulse vibrating method is proposed to solve the problem of small attachment force.

Our future researches include finding the principle of vibrating suction method from hydrodynamics and elasticity respects, analysing the unknown factors. Moreover, new mathematical models will be built and testified by the experimental platform. And the new attachment module will also be developed and compared with the old attachment module.

\section{REFERENCES}

[1] H.X. Zhang, J. Gonzalez-Gomez, S.Y. Chen, W. Wang, R. Liu, D.Z. $\mathrm{Li}$, and J.W. Zhang, "A Novel Module Climbing Caterpillar Using Low-frequency Vibrating Passive Suckers", Proceeding of 2007 IEEE/ASME International Conference on Advanced Intelligent Mechatronics, ETH Zurich, Switzerland, pp.85-90, Sept. 2007

[2] S.Q. Wu, M.T. Li, S. Xiao, and Y. Li, "A Wireless Distributed Wall Climbing Robotic System for Reconnaissance Purpose", Proceedings of the 2006 IEEE International Conference on Mechatronics and Automation, Luoyang, China, pp.1380-1312, June 2006.

[3] W. Shen, J. Gu, and Y. Shen, "Permanent Magnetic System Design for the Wall-climbing Robot," Proceedings of the IEEE International Conference on Mechatronics \& Automation, vol. 4, pp. 2078-2083, July 29-Aug. 1.2005.

[4] W. Shen, J. Gu, and Y. Shen, "Proposed Wall Climbing Robot with Permanent Magnetic Tracks for Inspecting Oil Tanks," Proceedings of the IEEE International Conference on Mechatronics \& Automation, vol. 4,pp. 2072-2077, July 29-Aug. 1, 2005.

[5] K.A. Daltorio, A.D. Horchler, S. Gorb, "A small wall-walking robot with compliant, adhesive feet," Proceedings of IEEE/RSJ International Conference on Intelligent Robots and Systems, 2005, pp. 36483653,Aug. 2005.

[6] M. Sitti, R.S. Fearing, "Synthetic gecko foot-robots," Proceedings of IEEE International Conference on Robotics and Automation, 2003, vol. 1,pp. 1164-1170, Sept. 2003.

[7] H.X. Zhang, J.W. Zhang, R. Liu, and G.H. Zong, "Realization of a service robot for cleaning spherical surfaces", International Journal of Advanced Robotic Systems, Vol.2, No.1, pp. 53-58, 2005.

[8] S. Hirose, A. Nagakubo, and R. Toyama, "Machine That Can Walk and Climb on Floors, Walls and Ceilings", In Proceedings of the 5th International Conference on Advanced Robotics, pp.753-758, 1991.

[9] $\mathrm{Na}$ Shun $\mathrm{Bu} \mathrm{He}$, Rong Liu, Wei Wang, Hao Yang and Xu Dong Wang , "A Mini Multi-joint Wall Climbing Robot Based on the Vibrating Suction Method" Proceedings of the 2007 IEEE International Conference on Robotics and Biomimetics December 15 -18, 2007, Sanya, China.

[10]Shanqiang Wu, Mantian Li, Shu Xiao and Yang Li, "A Wireless Distributed Wall Climbing Robotic System for Reconnaissance Purpose" Proceedings of the 2006 IEEE International Conference on Mechatronics and Automation June 25 - 28, 2006, Luoyang, Chin.
[11]Bing L. Luk, David S. Cooke, Stuart Galt, Arthur A. Collie, Sheng Chen, "Intelligent legged climbing service robot for remote maintenance applications in hazardous environments", Journal of Robotics and Autonomous System, ROBOT-1247, No.of pages 11, 2005.

[12]Tao Zhu Rong Liu, Xu D. Wang, Kun Wang, "Principle and Application of Vibrating Suction Method," s,2006.ROBIO'06 .IEEE International Conference on Robotics and Biomimetic, Dec. 2006 Page(s):491 - 495.

[13]Wei Wang, Yingying Wang, Jinhao Qi, Houxiang Zhang, Jianwei Zhang "The CPG Control Algorithm for a Climbing Worm Robot", Proceedings of the 3rd IEEE Conference on Industrial Electronics and Applications, Singapore, 3-5 June 2008, ISBN 978-1-4244-1718-6

[14]Wei Wang, Yingying Wang and Kun Wang, Houxiang Zhang, Jianwei Zhang, "Analysis of the Kinematics of Module Climbing Caterpillar Robots", Proceeding of 2008 IEEE/ASME International Conference on Advanced Intelligent Mechatronics, Xi'an, China, 2-5 July. 2008

[15]Jihong Liu, K. Tanaka, L.M. Bao, I. Yamaura, "Analytical modelling of suction cups used for window-cleaning robots", Journal of VACUUM, Vacuum 80 (2006) 593-598. 